\title{
ANTIMICROBIAL AND CHITINASE INHIBITORY ACTIVITIES OF 7-BENZAMIDOCOUMARINS
}

\author{
NEHA TIWARI ${ }^{1}$, PRIYANKA ${ }^{1}$, VINEETA SINGH ${ }^{2}$, DIKSHA KATIYAR ${ }^{1 *}$
}

${ }^{1}$ Department of Chemistry, Mahila Maha Vidyalaya, Banaras Hindu University, Varanasi, Uttar Pradesh, India. ${ }^{2}$ Department of Biotechnology, Institute of Engineering and Technology, Sitapur Road, Lucknow, Uttar Pradesh, India. Email: dikshakatiyar@gmail.com

Received: 20 March 2019, Revised and Accepted: 13 May 2019

ABSTRACT

Objective: The aim of this study was to examine in vitro antimicrobial activity of a series of 7-benzamidocoumarin derivatives against three Grampositive bacteria (Bacillus subtilis, Staphylococcus aureus, and Bacillus pumilus), three Gram-negative bacteria (Escherichia coli, Klebsiella pneumonia, and Salmonella typhi), and three fungi (Candida albicans, Candida tropicalis, and Aspergillus niger) by 2-fold serial dilution method.

Materials and Methods: The compounds were synthesized by amide coupling of 7-amino-4-methyl coumarin 5 and 7-amino-4-chloromethyl coumarin 6 with different aromatic acids 7 (a-h) and heteroaromatic acids 8 (i-j) in the presence of phosphorous trichloride as an acid catalyst in acetonitrile. The synthesized compounds were evaluated for antimicrobial and chitinase inhibitory activity.

Results: Compounds 9, 11, 12, 21, and 23 showed good antibacterial activity with minimum inhibitory concentration (MIC) ranging from 6.25 to $50 \mu \mathrm{g} / \mathrm{mL}$. Among them, compound 21 was the most active molecule with MIC $6.25 \mu \mathrm{g} / \mathrm{mL}$ against $S$. aureus and $6.25 \mu \mathrm{g} / \mathrm{mL}$ against, $B$. pumilus and S. typhi. Compounds 11 and 21 were the most potent antifungal candidates with MIC 6.25-25 $\mu \mathrm{g} / \mathrm{mL}$ against $C$. albicans and C. tropicalis. All the compounds were also evaluated for their chitinase inhibitory property and among them; compound 9 emerged as strong inhibitor of the enzyme.

Conclusion: Some of the compounds showed very good antimicrobial activities. Among these, compound 21 showing potent antimicrobial activities against five of the nine microbial strains examined in this study, was the most active compound of the series. Some of the compounds also showed chitinase inhibitory properties. This study also provides a starting point for investigating the structure-activity relationships (SARs) of synthetic 7-benzamidocoumarins.

Keywords: Amidocoumarins, Coumarin, Antimicrobial, Antibacterial, Antifungal, Chitinase.

(C) 2019 The Authors. Published by Innovare Academic Sciences Pvt Ltd. This is an open access article under the CC BY license (http://creativecommons. org/licenses/by/4. 0/) DOI: http://dx.doi.org/10.22159/ajpcr.2019.v12i7.33155

\section{INTRODUCTION}

Over the past decade, the incidence of bacterial infections has increased worldwide, particularly in Indian subcontinent, portions of South America, and tropical fraction of Africa [1,2]. In spite of the availability of a large number of antibiotics and chemotherapeutics, the treatment of bacterial diseases still remains a challenging problem due to a number of factors such as the emergence of drug-resistant strains, severe adverse effects, narrow antibacterial spectrum, and association of microbial infections with other diseases mainly in immunocompromised patients [3-5]. Despite the major cause of morbidity and mortality, these infections are given low priority, and most importantly, the pipeline for new antibiotics has paradoxically experienced a long-term decline [6].

Fungal infections also pose a continuous and serious threat to human health and life. It is estimated that about 1.2 billion people worldwide suffer from fungal diseases [7]. The opportunistic fungal pathogens belonging to Candida spp., Cryptococcus spp., and Aspergillus spp. account for the majority of documented invasive fungal infections in humans. These infections are responsible for high morbidity and mortality in immune-compromised patients such as those undergoing organ transplants or anticancer chemotherapy and patients with AIDS [8]. The existing mainstay antifungal drugs such as fluoroquinolones, amphotericin-B (Amp), and azoles are associated with a number of severe side effects and development of resistance [9]. Thus, there is a pressing need for the development of alternative antimicrobial drugs involving new molecules with a broader spectrum and less side effects.

Coumarins are a group of oxygen-containing heterocyclic compounds with a benzoannelated $\alpha$-pyrone ring. They are found in a large number of bioactive natural and non-natural molecules. Due to structure variability, low toxicity, and low cost, coumarins play an important role in modern drug discovery and synthetic chemistry. Coumarin derivatives display a wide range of biological activities such as antimalarial, antimicrobial, anticancer, anti-inflammatory, antioxidant, antiviral, antitubercular, and antifilarial $[10,11]$. The pharmacological, biochemical, and therapeutical potential of coumarin compounds mainly depend on the substituent they bear on their basic chemical skeleton [12]. Another, unique feature of coumarin, which makes it a privileged structural framework, is its ability to interact readily with a diversity of enzymes and receptors in organisms through weak bond interactions. Therefore, substitution on the lactone and aromatic rings of a coumarin motif provides opportunities for modulation of biological activities and enhancement in pharmacokinetic properties. In recent years, coumarin derivatives have gained tremendous attention due to their applications in drug design and discovery $[13,14]$. Keeping in view of the above and in continuation of our work on the design and synthesis of coumarin compounds possessing antimicrobial and antifilarial properties $[15,16]$, we report herein the synthesis and antimicrobial activity of a series of 7-benzamidocoumarins. The synthesized compounds were also evaluated for their chitinase inhibitory activity. To carry out this study, first, we screened six simple coumarin scaffolds for their antimicrobial properties. Out of these, the 4-methyl-and 4-chloromethyl-7-aminocoumarins which showed the best activity were chosen as starting material for further optimization study. We hope this study may be useful in further design of coumarinbased molecules as antimicrobial drugs or prodrugs.

\section{MATERIALS AND METHODS}

Unless otherwise stated, all materials were obtained from commercial suppliers (SRL and Spectrochem Pvt., Ltd.) and were used without 
further purification. Melting points were determined on a Buchi 510 apparatus and are uncorrected. Silica gel (60-120 mesh) from commercial supplier Spectrochem Pvt., Ltd. was used for column chromatography. Thin-layer chromatography was used to monitor the reactions, and the spots were visualized by spraying $\mathrm{KMnO}_{4}$ reagent. IR spectra were recorded on JASCO FTIR 5300 in $\mathrm{KBr}$ from 400 to 4000 $\mathrm{cm}^{-1}$. NMR spectra were recorded on JEOL AL 300 FT-NMR spectrometer using tetramethylsilane as an internal standard. The chemical shift values are on $\delta$ scale and the coupling constant $(U)$ is in hertz (Hz).

\section{Synthetic procedure}

4-methyl-7-hydroxy-6-nitrocoumarin 4

4-Methyl-7-hydroxycoumarin $(250 \mathrm{mg}$ ) was dissolved in $2 \mathrm{~mL}$ of conc. sulfuric acid at room temperature and cooled at $0^{\circ} \mathrm{C}$. To this solution, a chilled nitrating mixture $(3 \mathrm{~mL}$ of concentrated nitric acid added to $9 \mathrm{~mL}$ of concentrated sulfuric acid) was added. The solution was stirred at $0^{\circ} \mathrm{C}$ for $3 \mathrm{~h}$ and then poured over crushed ice. The precipitated bright yellow product was filtered off, washed with ice cold water and recrystallized from ethanol to give compound 4 as yellow, green crystals [17].

\section{7-Amino-4-methyl-2H-chromen-2-one 5}

First, ethyl (3-hydroxyphenyl) carbamate was prepared by the following method: To stirred suspension of 3-aminophenol (5 g, $45.5 \mathrm{mmol})$ in $20 \mathrm{~mL}$ of anhydrous diethyl ether, ethyl chloroformate $(6 \mathrm{~g}, 54.6 \mathrm{mmol})$ was added. The reaction mixture was stirred for $2 \mathrm{~h}$ atroom temperature. After the completion of the reaction, the amine hydrochloride was removed by filtration. The filtrate was then evaporated to give off white solid, which was recrystallized from petroleum ether to afford carbamate derivative as white crystalline solid. To the stirred solution of the carbamate $(2.0 \mathrm{~g}, 11.0 \mathrm{mmol})$ in $25 \mathrm{~mL}$ of $70 \% \mathrm{H}_{2} \mathrm{SO}_{4}$ in ethanol (18 $\mathrm{mL} \mathrm{H}_{2} \mathrm{SO}_{4}+7 \mathrm{~mL}$ EtOH), ethyl acetoacetate $(2.18 \mathrm{~g}, 13.25 \mathrm{mmol})$ was added and the reaction mixture was stirred at room temperature for $4 \mathrm{~h}$. The mixture was then poured in $50 \mathrm{~mL}$ of ice cold water, giving ethyl (4-[methyl]-2-oxo-2H-chromen-7-yl) carbamate as white precipitate that was crystallized from absolute EtOH. Ethyl (4-chloromethyl-2-oxo-2H-chromen-7-yl) carbamate was also prepared by the same procedure using ethyl chloro acetoacetate.

In the next sequence of reaction, 7-carbethoxyamino-4-methyl coumarin $(1.5 \mathrm{~g}, 5.33 \mathrm{mmol})$ was dissolved in a solution of $10 \mathrm{~mL}$ of concentrated $\mathrm{H}_{2} \mathrm{SO}_{4}$ and $10 \mathrm{~mL}$ of glacial acetic acid. The mixture was heated at reflux for $2 \mathrm{~h}$. After cooling to room temperature, the mixture was poured in $50 \mathrm{~mL}$ of ice cold water. At $0^{\circ} \mathrm{C}, 50 \% \mathrm{NaOH}$ was added until the solution resulted slightly alkaline $(\mathrm{pH}=9-10)$. The obtained precipitate was filtered and washed with ice cold water $(10 \mathrm{~mL})$ to afford the desired 7-amino-4-methyl-2H-chromen-2-one $\mathbf{5}$ as light yellow solid $[18,19]$.

\section{7-amino-4-chloromethyl-2H-chromen-2-one (6)}

The reaction of 7-carbethoxyamino-4-chloromethyl coumarin $(2.5 \mathrm{~g}, 13.8 \mathrm{mmol})$ in the presence of $2 \mathrm{~mL}$ of concentrated $\mathrm{H}_{2} \mathrm{SO}_{4}$ and $2 \mathrm{~mL}$ of glacial acetic acid under reflux condition for a period of $2 \mathrm{~h}$ as described above gave 7-amino-4-chloromethyl-2H-chromen-2-one 6 as light brown solid.

\section{General procedure for the synthesis of 7-benzamidocoumarin} derivatives 9-23

Amino coumarins 5 and $6(1 \mathrm{mmol})$ and appropriate aromatic acids 7 (a-h) or hetero-aromatic acids $8(\mathrm{i}-\mathrm{j})(1.2 \mathrm{mmol})$ were dissolved in acetonitrile $(5 \mathrm{~mL}), \mathrm{PCl}_{3}(1 \mathrm{mmol})$ and the reaction mixture was refluxed for 3-6 h. After completion of the reaction, the reaction mixture was quenched with a few drops of water. The solvent was evaporated; the residue was dissolved in ethyl acetate $(10 \mathrm{~mL})$ and washed twice with saturated $\mathrm{NaHCO}_{3}$ solution. The organic layer was dried over $\mathrm{Na}_{2} \mathrm{SO}_{4}$ and the solvent was evaporated under reduced pressure. The crude product was purified by column chromatography on silica gel using $2 \%$ $\mathrm{CH}_{3} \mathrm{OH}$ in $\mathrm{CHCl}_{3}$ solvent system as mobile phase [20].

\section{Determination of MIC}

Synthesized compounds were evaluated on the basis of their antimicrobial activities examined in terms of MIC against six multiple drug-resistant bacterial test strains, namely B. subtilis MTCC 121, S. aureus MTCC 96, B. pumilus MTCC 1607, K. pneumoniae MTCC 3384, S. typhi MTCC 537, and E. coli MTCC1304 and three fungi C. albicans MTCC 3017, C. tropicalis MTCC 184, and A. niger MTCC 1344. MIC is the lowest concentration of the compound that will be able to inhibit the microbial growth under experimental conditions. Erythromycin (Ery) and Amp were used as standards for antibacterial and antifungal activity, respectively.

MIC of the synthesized compounds was determined by the micro broth dilution method using 96 well plates according to the method described by Vipra et al. [21] with some modification. The bacterial test organisms were grown in sterile nutrient broth (NB) at $30^{\circ} \mathrm{C}$. From this stock solution certain inoculums were used to inoculate fresh medium, and the final inoculum size for test strains was adjusted to $10^{6} \mathrm{cfu} / \mathrm{mL}$ (colony-forming unit per milliliter). Fresh NB was taken as a negative control in the first well, whereas NB with test organisms were consider as a positive control in the last well. Stock solutions of $1 \mathrm{mg} / \mathrm{mL}$ of compounds were prepared. From this, $100 \mu \mathrm{g} / \mathrm{mL}$ concentrations were taken as starting solution in the second well and diluted serially up to the second last well. Microtiter plates were incubated at $30^{\circ} \mathrm{C}$ for overnight under the static condition and read using ELISA plate reader. Well containing the lowest concentration of the compounds at which no turbidity was observed consider as MIC.

\section{Chitinase assay for coumarin derivatives}

Chitinase inhibitory activity of the coumarin compounds was determined by Dinitrosalicylic acid (DNS) as mentioned by Miller using colloidal chitin as a substrate [22]. Colloidal chitin was prepared from the chitin powder. Five grams of chitin powder $\left(\mathrm{C}_{8} \mathrm{H}_{13} \mathrm{O}_{5} \mathrm{~N}\right)$ were gradually mixed with $6 \mathrm{~mL}$ concentrated $\mathrm{HCl}(32 \%)$ and incubated at room temperature with vigorous stirring for about $2 \mathrm{~h}$ on an orbital shaker. Ice-cold water was added to precipitate the chitin and this solution was further incubated overnight. After incubation, it was centrifuged at $10,000 \mathrm{rpm}$ for $20 \mathrm{~min}$ at $4^{\circ} \mathrm{C}$. The pellet was washed repeatedly with distilled water until the $\mathrm{pH}$ of the chitin suspension reached the $\mathrm{pH}$ of distilled water. Finally, the substrate was autoclaved and stored at $4^{\circ} \mathrm{C}$.

Reaction mixture containing $100 \mu \mathrm{l}$ of coumarin compound (stock solution $1 \mathrm{mg} / \mathrm{ml}$ ), $1 \mathrm{ml}$ colloidal chitin and $1 \mathrm{ml}$ chitinase extracted from actinomycetes strain were taken in test tubes and incubated for $30 \mathrm{~min}$ at $37^{\circ} \mathrm{C}$ in shaking water bath. The reaction was stopped by adding $2 \mathrm{ml}$ DNS reagent and kept in a boiling water bath for $5 \mathrm{~min}$ to develop the color. After cooling the test tubes for $10 \mathrm{~min}$; color was observed against control. The concept lies in DNS reaction is the change in color from yellowish orange to brownish orange.

\section{RESULTS AND DISCUSSION}

\section{Chemistry}

Compounds 1-3 were purchased from the commercial supplier and used without further purification. Compound 4 was synthesized by nitration of 4-methyl-7-hydroxycoumarin under cold condition by earlier reported method [17]. Compounds 5 and 6 were prepared by following method. In the first step, protected amino phenol was synthesized by reaction of 3-amino phenol with ethylchloroformate in presence of $\mathrm{K}_{2} \mathrm{CO}_{3}$. The protected amino phenol on Pechmann condensation with ethyl acetoacetate and ethyl 4-chloroacetoacetate in the presence of $70 \%$ ethanolic $\mathrm{H}_{2} \mathrm{SO}_{4}$ at room temperature followed by deprotection of carbamate group using mixture of $\mathrm{H}_{2} \mathrm{SO}_{4}$ and $\mathrm{CH}_{3} \mathrm{COOH}$ (1:1) afforded 7-amino-4-methyl coumarin 5 and 7-amino-4chloromethyl coumarin 6 , respectively, in good yields $[18,19]$. With the desired amino coumarins 5 and 6 in hand, the synthesis of 7-benzamidecoumarins was undertaken. 7-benzamido-4-methyl-coumarins 9-11 were obtained by reaction between 7-amino-4-methyl coumarin 5 and 
substituted salicylic acids 7 a-c in $\mathrm{CH}_{3} \mathrm{CN}$ in the presence of $\mathrm{PCl}_{3}$ under reflux condition while compounds $12-14$ and 15 were prepared by the reaction of 7-amino-4-chloromethyl coumarin 6 with substituted salicylic acids $7 \mathrm{a}-\mathrm{c}$ and unsubstituted salicylic acid $7 \mathrm{~d}$, respectively. Further, the synthesis of amidocoumarins 16-19 was carried out by reaction between compound 6 and benzoic acid derivatives 7 (e-h) bearing electron releasing and electron withdrawing groups as described above (Scheme 1). In the next sequence of reactions, few heteroaromatic acids such as 2-picolinic acid 8i and 2-pyrazinoic acid 8j were selected to synthesize 7-amidocoumarins 20-23 (Scheme 1). The spectroscopic data of all the compounds reported herein were found to be in good agreement with previously published data [20]. As a representative example, the structure elucidation of compound 19 , obtained by the reaction between 7-amino-4-chloromethyl coumarin 6 and 2-methyl benzoic acid $7 \mathrm{~h}$ is described here. The compound exhibited absorption bands at 3331 and $1714 \mathrm{~cm}^{-1}$ in the IR spectrum for $\mathrm{N}-\mathrm{H}$ stretching and $\delta$-lactone, respectively. In ${ }^{1} \mathrm{H}$ NMR spectrum, $-\mathrm{NH}$ proton appeared at $\delta 10.3$ as broad singlet while signal corresponding to H-6' proton of benzamide ring was observed as multiplet at $\delta 7.94$. Doublet appeared at $\delta 7.81$ with coupling constant of $2.4 \mathrm{~Hz}$ was due to the H-8 proton of the benzamide ring, whereas doublets at $\delta 7.61$ and $\delta 7.50$ with a coupling constant of $8.7 \mathrm{~Hz}$ were due to the H-5 and H-6 protons of benzopyrone ring, respectively. Signal due to H-4' was found as multiplet at $\delta 7.38$ while multiplet at $\delta 7.29$ was ascribed to the $\mathrm{H}-3^{\prime}$ and $\mathrm{H}-5^{\prime}$ protons of the benzamide ring. Singlets at $\delta 6.45,4.69$, and 2.38 were assigned to $\mathrm{H}-3$ of lactone ring, methylene protons $(-\mathrm{CH} C \mathrm{Cl}$, and methyl group at C-2' carbon, respectively.

\section{4-Methyl-7-hydroxy-6-nitrocoumarin (4)}

Yield: $78 \%$; mp: $195^{\circ} \mathrm{C}-196^{\circ} \mathrm{C}$; IR (KBr): $3125,3060,1730,1620,1530$, $1390 \mathrm{~cm}^{-1} .{ }^{1} \mathrm{H}$ NMR (DMSO-d, $300 \mathrm{MHz}$ ): $\delta 2.38\left(\mathrm{~s}, 3 \mathrm{H}, \mathrm{CH}_{3}\right.$ ), $6.41(\mathrm{~s}, 1 \mathrm{H})$, $7.40(\mathrm{~s}, 1 \mathrm{H}), 8.48(\mathrm{~s}, 1 \mathrm{H})$.

\section{7-Amino-4-methyl-2H-chromen-2-one (5)}

$71 \%$; mp $223^{\circ} \mathrm{C}-224^{\circ} \mathrm{C}$ (lit. mp: $220^{\circ} \mathrm{C}-222^{\circ} \mathrm{C}^{18}$ ); ${ }^{1} \mathrm{H}$ NMR (DMSO-d, $400 \mathrm{MHz}) \delta: 2.39$ (s, 3H), $5.92(\mathrm{~s}, 1 \mathrm{H}), 6.10$ (brs, 2H), 6.37 (s, 1H), 6.58 $(\mathrm{m}, 1 \mathrm{H}), 7.36(\mathrm{~d}, J=8.8 \mathrm{~Hz}, 1 \mathrm{H})$.

\section{7-amino-4-chloromethyl-2H-chromen-2-one (6)}

Yield: 70\%; ${ }^{1} \mathrm{H}$ NMR (DMSO-d, $\left.300 \mathrm{MHz}\right) \delta: 4.85(\mathrm{~s}, 2 \mathrm{H}), 6.16(\mathrm{~s}, 1 \mathrm{H})$, $6.22(\mathrm{~s}, 2 \mathrm{H}), 6.40(\mathrm{~d}, J=2.2 \mathrm{~Hz}, 1 \mathrm{H}), 6.58(\mathrm{dd}, J=8.8$ and $2.2 \mathrm{~Hz}, 1 \mathrm{H})$, $7.47(\mathrm{~d}, J=8.8 \mathrm{~Hz}, 1 \mathrm{H})$.

2-hydroxy-N-(4-methyl-2-oxo-2H-chromen-7-yl)-3,5-dinitrobenzamide (9) Light yellow solid; yield: $75 \%$; mp: $153-154{ }^{\circ} \mathrm{C}$; IR $\left(\mathrm{cm}^{-1}, \mathrm{KBr}\right) v_{\max }$ : 3323, 1718, 1532, 1356; ${ }^{1} \mathrm{H}$ NMR (DMSO- $\left.\mathrm{d}_{6}, 500 \mathrm{MHz}\right) \delta: 2.36$ (s, 3H,
$\mathrm{CH}_{3}$ ), $6.46(\mathrm{~s}, 1 \mathrm{H}), 7.25(\mathrm{~d}, J=1.5 \mathrm{~Hz}, 1 \mathrm{H}), 7.88(\mathrm{~d}, J=8.5 \mathrm{~Hz}, 1 \mathrm{H}), 7.93$ $(\mathrm{m}, 2 \mathrm{H}), 8.57(\mathrm{~s}, 1 \mathrm{H}), 9.65(\mathrm{~s}, 1 \mathrm{H})$.

2-hydroxy-3,5-diiodo-N-(4-methyl-2-oxo-2H-chromen-7-yl) benzamide (10) Off white solid; yield: $79 \%$; mp: $176^{\circ} \mathrm{C}-178^{\circ} \mathrm{C}$; IR $\left(\mathrm{cm}^{-1}, \mathrm{KBr}\right) v_{\max }: 3341$, 1708, 1528, 1349; ${ }^{1} \mathrm{H}$ NMR (DMSO-d, $\left.500 \mathrm{MHz}\right) \delta: 2.38\left(\mathrm{~s}, 3 \mathrm{H}, \mathrm{CH}_{3}\right.$ ), $6.18(\mathrm{~s}, 1 \mathrm{H}, \mathrm{H}-3), 7.36(\mathrm{~d}, J=9.0 \mathrm{~Hz}, 1 \mathrm{H}), 7.67(\mathrm{~d}, J=8.5 \mathrm{~Hz}, 1 \mathrm{H}), 7.73$ $(\mathrm{d}, J=2.0 \mathrm{~Hz}, 1 \mathrm{H}), 7.92(\mathrm{~s}, 1 \mathrm{H}), 7.95(\mathrm{~s}, 1 \mathrm{H})$.

3,5-dibromo-2-hydroxy-N-(4-methyl-2-oxo-2H-chromen-7-yl) benzamide (11)

Off white solid; yield: $80 \%$; $\mathrm{mp}: 165^{\circ} \mathrm{C}-167^{\circ} \mathrm{C}$; IR $\left(\mathrm{cm}^{-1}, \mathrm{KBr}\right) v_{\text {max }}: 3366$, $1700,1534,1348 ;{ }^{1} \mathrm{H}$ NMR (DMSO-d $\left.{ }_{6}, 500 \mathrm{MHz}\right) \delta: 2.38(\mathrm{~s}, 3 \mathrm{H}), 6.18$ (s, $1 \mathrm{H}), 7.34(\mathrm{~d}, J=8.5 \mathrm{~Hz}, 1 \mathrm{H}), 7.66(\mathrm{~d}, J=8.5 \mathrm{~Hz}, 1 \mathrm{H}), 7.81(\mathrm{~m}, 2 \mathrm{H}), 7.95$ $(\mathrm{s}, 1 \mathrm{H}), 15.21(\mathrm{~s}, 1 \mathrm{H})$.

$N$-(4-[chloromethyl]-2-oxo-2H-chromen-7-yl)-2-hydroxy-3,5-dinitro benzamide (12)

Yellow solid; yield: $78 \%$; mp: $140^{\circ} \mathrm{C}-142^{\circ} \mathrm{C}$; IR $\left(\mathrm{cm}^{-1}, \mathrm{KBr}\right) v_{\max }: 3343$, 1711, 1569, 1349; ${ }^{1} \mathrm{H}$ NMR (DMSO-d, $\left.500 \mathrm{MHz}\right) \delta: 4.95(\mathrm{~s}, 2 \mathrm{H}$ ), 6.72 $(\mathrm{s}, 1 \mathrm{H}), 7.47(\mathrm{~m}, 2 \mathrm{H}), 7.79(\mathrm{~s}, 1 \mathrm{H}), 8.02(\mathrm{~d}, J=2.0 \mathrm{~Hz}, 1 \mathrm{H}), 8.61(\mathrm{~s}, 1 \mathrm{H})$, $10.85(\mathrm{~s}, 1 \mathrm{H}, \mathrm{NH})$.

N-(4-[chloromethyl]-2-oxo-2H-chromen-7-yl)-2-hydroxy-3,5-diiodo benzamide (13)

Off white solid; yield: $80 \%$; mp: $169^{\circ} \mathrm{C}-170^{\circ} \mathrm{C}$; IR ( $\left.\mathrm{cm}^{-1}, \mathrm{KBr}\right) v_{\max }: 3331$, 1699, 1619, 1336; ${ }^{1} \mathrm{H}$ NMR (DMSO-d, $\left.500 \mathrm{MHz}\right) \delta: 4.96(\mathrm{~s}, 2 \mathrm{H}), 6.57$ $(\mathrm{s}, 1 \mathrm{H}), 7.71(\mathrm{~d}, J=2.0 \mathrm{~Hz}, 1 \mathrm{H}), 7.80(\mathrm{~m}, 2 \mathrm{H}), 8.20(\mathrm{~m}, 2 \mathrm{H})$.

3,5-dibromo-N-(4-[chloromethyl]-2-oxo-2H-chromen-7-yl)-2-hydroxy benzamide (14)

Off white solid; yield: $79 \%$; $\mathrm{mp}: 166^{\circ} \mathrm{C}-168^{\circ} \mathrm{C}$; IR $\left(\mathrm{cm}^{-1}, \mathrm{KBr}\right) v$ : 3314 , 1701, 1528, 1345; ${ }^{1} \mathrm{H}$ NMR (DMSO-d $\left.6,500 \mathrm{MHz}\right) \delta: 4.94(\mathrm{~s}, 2 \mathrm{H}), 6.53$ $(\mathrm{s}, 1 \mathrm{H}), 7.54(\mathrm{dd}, J=8.5$ and $2.0 \mathrm{~Hz}, 1 \mathrm{H}), 7.68(\mathrm{~d}, J=2.0 \mathrm{~Hz}, 1 \mathrm{H}), 7.72$ (d, $J=8.5 \mathrm{~Hz}, 1 \mathrm{H}$ ), 8.34 (d, $J=2.0 \mathrm{~Hz}, 1 \mathrm{H}), 8.46$ (d, $J=2.0 \mathrm{~Hz}, 1 \mathrm{H}), 10.85$ (s, 1H).

$\mathrm{N}$-(4-[chloromethyl]-2-oxo-2H-chromen-7-yl)-2-hydroxybenzamide (15) Colorless solid; yield: $82 \%$; mp: $146^{\circ} \mathrm{C}-147^{\circ} \mathrm{C}$; IR $\left(\mathrm{cm}^{-1}, \mathrm{KBr}\right) v_{\text {ax }}: 3371$, 1728, 1578, 1228; ${ }^{1} \mathrm{H}$ NMR DMSO-d $\left.600 \mathrm{MHz}\right) \delta$ : 4.96(s, 2H), 6.54 $(\mathrm{s}, 1 \mathrm{H}), 6.94(\mathrm{~m}, 2 \mathrm{H}), 7.39(\mathrm{~m}, 1 \mathrm{H}), 7.67(\mathrm{~m}, 2 \mathrm{H}), 7.89(\mathrm{~m}, 2 \mathrm{H}), 10.84(\mathrm{~s}, 1 \mathrm{H})$.

$\mathrm{N}$-(4-[chloromethyl]-2-oxo-2H-chromen-7-yl) benzamide (16)

Colorless solid; yield: $81 \%$; mp: $137^{\circ} \mathrm{C}-139^{\circ} \mathrm{C}$; IR $\left(\mathrm{cm}^{-1}, \mathrm{KBr}\right) v_{\max }$ : 3431, 1722, 1594, 1338; ${ }^{1} \mathrm{H}$ NMR $\left(\mathrm{CDCl}_{3}, 300 \mathrm{MHz}\right) \delta: 4.60(\mathrm{~s}, 2 \mathrm{H}), 6.56$

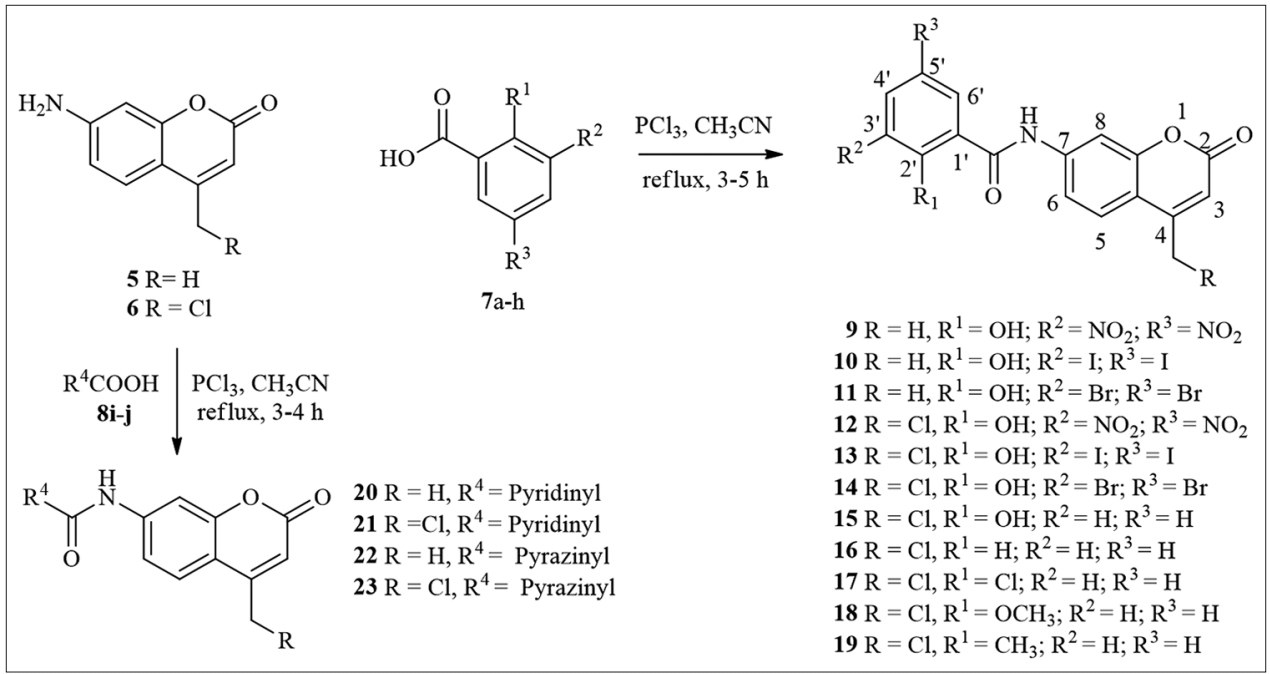

Scheme 1: Synthesis of 7-amidocoumarins 
(s, 1H), $7.16(\mathrm{~m}, 1 \mathrm{H}), 7.35(\mathrm{~m}, 2 \mathrm{H}), 7.47(\mathrm{~m}, 1 \mathrm{H}), 7.66(\mathrm{~m}, 1 \mathrm{H}), 7.71$ $(\mathrm{m}, 3 \mathrm{H})$.

2-chloro- $\mathrm{N}$-(4-[chloromethyl]-2-oxo-2H-chromen-7-yl) benzamide (17) Colorless solid; yield: $81 \%$; mp: $146^{\circ} \mathrm{C}-147^{\circ} \mathrm{C}$; IR ( $\left.\mathrm{cm}^{-1}, \mathrm{KBr}\right) v_{\text {max }}: 3421$, $1732,1591,1261 ;{ }^{1} \mathrm{H}$ NMR $\left(\mathrm{CDCl}_{3}, 300 \mathrm{MHz}\right) \delta: 4.62(\mathrm{~s}, 2 \mathrm{H}), 6.60(\mathrm{~s}, 1 \mathrm{H})$, $7.19(\mathrm{~m}, 2 \mathrm{H}), 7.41(\mathrm{~m}, 4 \mathrm{H}), 7.69(\mathrm{~d}, J=8.4 \mathrm{~Hz}, 1 \mathrm{H})$.

$\mathrm{N}$-(4-[chloromethyl]-2-oxo-2H-chromen-7-yl)-2-methoxybenzamide (18) Colorless solid; yield: $80 \%$; mp: $160^{\circ} \mathrm{C}-162^{\circ} \mathrm{C}$; IR ( $\left.\mathrm{cm}^{-1}, \mathrm{KBr}\right) v_{\text {max }}: 3317$, 1726, 1587, 1298; ${ }^{1} \mathrm{H} \mathrm{NMR}\left(\mathrm{CDCl}_{3}, 300 \mathrm{MHz}\right) \delta: 3.18$ (s, 3H), 4.27(s, 2H), $5.86(\mathrm{~s}, 1 \mathrm{H}), 6.37(\mathrm{~m} 1 \mathrm{H}), 6.50(\mathrm{~m}, 1 \mathrm{H}), 6.82(\mathrm{~m}, 1 \mathrm{H}), 6.94(\mathrm{~m}, 2 \mathrm{H}), 7.12$ $(\mathrm{d}, J=8.5 \mathrm{~Hz}, 1 \mathrm{H}), 7.24(\mathrm{~m} \mathrm{1H}), 9.87(\mathrm{~s}, 1 \mathrm{H})$.

$N$-(4-[chloromethyl]-2-oxo-2H-chromen-7-yl)-2-methylbenzamide (19) Colorless solid; yield: $84 \%$; mp: $149^{\circ} \mathrm{C}-151^{\circ} \mathrm{C}$; IR $\left(\mathrm{cm}^{-1}, \mathrm{KBr}\right) v_{\max }: 3331$, $1713,1582,1250 ;{ }^{1} \mathrm{H}$ NMR $\left(\mathrm{CDCl}_{3}, 300 \mathrm{MHz}\right) \delta: 2.38(\mathrm{~s}, 3 \mathrm{H}), 4.69(\mathrm{~s}, 2 \mathrm{H})$, 6.45 (s, 1H), $7.29(\mathrm{~m}, 2 \mathrm{H}), 7.38(\mathrm{~m}, 1 \mathrm{H}), 7.50(\mathrm{~d}, J=8.7 \mathrm{~Hz}, 1 \mathrm{H}), 7.61$ (d, J = 8.7 Hz, 1H), 7.81 (d, J = 2.4 Hz, 1H), $7.94(\mathrm{~m}, 1 \mathrm{H}), 10.3(\mathrm{~s}, 1 \mathrm{H})$.

\section{$\mathrm{N}$-(4-methyl-2-oxo-2H-chromen-7-yl) picolinamide (20)}

Colorless solid; Yield: $83 \%$; mp: $180^{\circ} \mathrm{C}-182^{\circ} \mathrm{C}$; IR $\left(\mathrm{cm}^{-1}, \mathrm{KBr}\right) v_{\max }: 3324$, $1713,1572,1323 ;{ }^{1} \mathrm{H}$ NMR (DMSO-d, $\left.500 \mathrm{MHz}\right) \delta: 2.36\left(\mathrm{~s}, 3 \mathrm{H}, \mathrm{CH}_{3}\right.$ ), 5.32 $(\mathrm{s}, 1 \mathrm{H}, \mathrm{H}-3), 6.78(\mathrm{~m}, 1 \mathrm{H}), 6.78(\mathrm{~d}, J=8.5 \mathrm{~Hz}, 1 \mathrm{H}), 6.93(\mathrm{dd}, J=8.5$ and $2.0 \mathrm{~Hz}, 1 \mathrm{H}), 7.17(\mathrm{~m}, 1 \mathrm{H}), 7.29(\mathrm{~m}, 2 \mathrm{H}), 7.86(\mathrm{~d}, J=4.5 \mathrm{~Hz}, 1 \mathrm{H}), 9.84$ (s, 1H, NH).

$\mathrm{N}$-(4-[chloromethyl]-2-oxo-2H-chromen-7-yl) picolinamide (21) Off white solid; yield: $82 \%$; mp: $184^{\circ} \mathrm{C}-186^{\circ} \mathrm{C}$; IR $\left(\mathrm{cm}^{-1}, \mathrm{KBr}\right) v_{\max }$ : 3329, 1745, 1623, 1332; ${ }^{1} \mathrm{H}$ NMR (DMSO-d, $\left.500 \mathrm{MHz}\right) \delta: 4.95$ (s, 2H), $6.54(\mathrm{~s}, 1 \mathrm{H}), 7.68(\mathrm{~m}, 1 \mathrm{H}), 7.79(\mathrm{~d}, J=9.0 \mathrm{~Hz}, 1 \mathrm{H}), 7.92(\mathrm{~d}, J=9.0 \mathrm{~Hz}, 1 \mathrm{H})$, $8.1(\mathrm{~s}, 1 \mathrm{H}), 8.09(\mathrm{~m}, 1 \mathrm{H}), 8.17(\mathrm{~d}, J=7.5 \mathrm{~Hz}, 1 \mathrm{H}), 8.72(\mathrm{~d}, J=4.0 \mathrm{~Hz}, 1 \mathrm{H})$, $11.05(\mathrm{~s}, 1 \mathrm{H}, \mathrm{NH})$.

N-(4-methyl-2-oxo-2H-chromen-7-yl) pyrazine-2-carboxamide (22) Light yellow solid; yield: $81 \%$; mp: $170^{\circ} \mathrm{C}-171^{\circ} \mathrm{C}$; IR $\left(\mathrm{cm}^{-1}, \mathrm{KBr}\right) v_{\max }$ : 3428, 1710, 1615, 1391; ${ }^{1} \mathrm{H}$ NMR (DMSO-d, $\left.300 \mathrm{MHz}\right) \delta: 2.39$ (s, 3H), $6.23(\mathrm{~s}, 1 \mathrm{H}), 7.64(\mathrm{~m}, 1 \mathrm{H}), 7.75(\mathrm{~m}, 1 \mathrm{H}), 7.96(\mathrm{~s}, 1 \mathrm{H}), 8.69(\mathrm{~s}, 1 \mathrm{H}), 8.84$ $(\mathrm{s}, 1 \mathrm{H}), 9.46(\mathrm{~s}, 1 \mathrm{H}), 10.21(\mathrm{~s}, 1 \mathrm{H})$.

$\mathrm{N}$-(4-[chloromethyl]-2-oxo-2H-chromen-7-yl) pyrazine-2-carboxamide (23) Off white solid; yield: $81 \%$; mp: $179^{\circ} \mathrm{C}-180^{\circ} \mathrm{C}$; IR ( $\left.\mathrm{cm}^{-1}, \mathrm{KBr}\right) v_{\max }: 3304$, 1708, 1617, 1332; ${ }^{1} \mathrm{H}$ NMR (DMSO-d, $\left.500 \mathrm{MHz}\right) \delta: 4.94(\mathrm{~s}, 2 \mathrm{H}), 6.54$ $(\mathrm{s}, 1 \mathrm{H}), 7.86(\mathrm{~m}, 2 \mathrm{H}), 8.1(\mathrm{~d}, J=1.5 \mathrm{~Hz}, 1 \mathrm{H}), 8.82(\mathrm{~d}, J=2.0 \mathrm{~Hz}, 1 \mathrm{H}), 8.91$ $(\mathrm{d}, J=2.0 \mathrm{~Hz}, 1 \mathrm{H}), 9.28(\mathrm{~d}, J=2.0 \mathrm{~Hz}, 1 \mathrm{H}), 11.2(\mathrm{~s}, 1 \mathrm{H})$.

\section{Antimicrobial activity}

First, we evaluated six simple coumarins 1-6 against three Grampositive bacterial strains B. subtilis MTCC 121(Bs), S. aureus MTCC 96 (Sa), and B. pumilus MTCC1607 (Bp) and three Gram-negative strains E. coli MTCC1304 (Ec), K. pneumoniae MTCC 3384(Kp), and S. typhi MTCC 537 (St) to determine the best skeleton for further modification. Out of these compounds, aminocoumarins 5 and 6 exhibited superior activity against the tested strains as compared to compounds 1-4 (Table 1). Therefore, compounds 5 and 6 were selected for further molecular optimization. Using amino coumarins 5 and 6 as basic skeleton, 7-amidocoumarins 9-23 were synthesized and examined for their MIC by the two-fold serial microdilution method using clinically antibacterial drug Ery standard [23] against the same above stated bacterial strains. The results are shown in Table 2 and Figs. 1 and 2 . The MIC was defined as the lowest concentration of the tested compound at which no growth of the strain was observed in a period of time and under specified experimental conditions.

As shown in Table 2, among the 4-methyl series, compounds 9 and 11 bearing dinitro and dibromo substituted salicylic acid residue, respectively, showed MIC $12.5 \mu \mathrm{g} / \mathrm{mL}$ against $B$. pumilus and $K$.

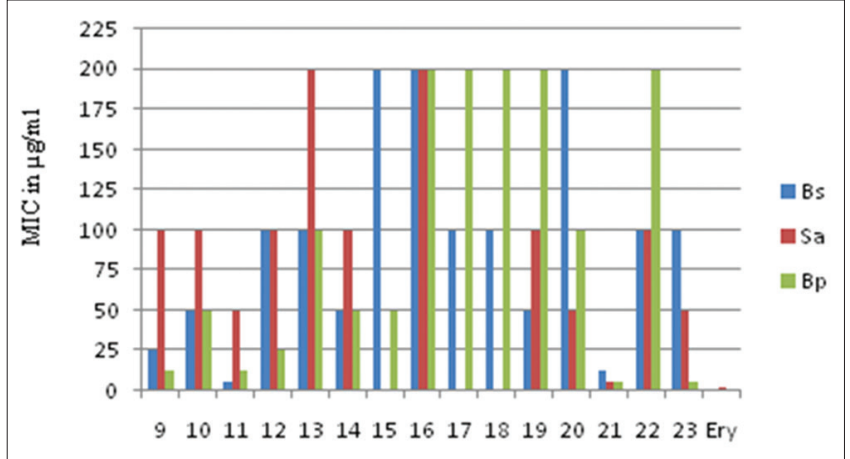

Fig. 1: Antibacterial activity of amidocoumarins against Grampositive bacteria

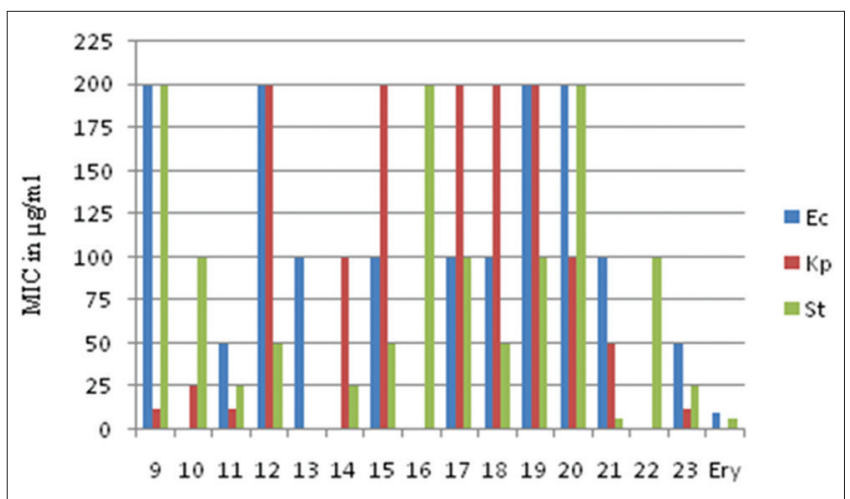

Fig. 2: Antibacterial activity of amidocoumarins against Gramnegative bacteria

pneumonia. Compound 11 showed superior activity against B. subtilis $(6.25 \mu \mathrm{g} / \mathrm{mL})$ as compared to compound $9(25 \mu \mathrm{g} / \mathrm{mL})$. Compounds $12-$ 15 bearing 4-chloromethyl group showed low order of activity against all the tested strain with MIC's in the range of $25-200 \mu \mathrm{g} / \mathrm{mL}$. Further, compounds 16-19 bearing unsubstituted benzoic acid, chloro, methoxy, and methyl substituted benzoic acid residue, respectively, showed inferior activity or even no inhibition against all the tested bacterial strains with MIC values in the range of 50 to $>200 \mu \mathrm{g} / \mathrm{mL}$. Further, the role of salicylic acid residue was explored with the synthesis and compounds 20-23 bearing heteroaromatic acid substituent. The activity was improved with the introduction of pyridinyl and piperazinyl ring, but surprisingly, in this case, 4-chloromethyl group bearing compounds exhibited superior activity than the 4-methyl group-containing compounds ( 21 and 23 vs. 20 and 22). Compound 21 bearing 4-chloromethyl and pyridinyl substituent showed superior activity among all the tested compounds and was found to be the most active compound of the series. It showed activity against four bacterial strains with MIC ranging from 6.25 to $25 \mu \mathrm{g} / \mathrm{mL}$. Its potency against $S$. typhi was comparable to the standard drug Ery. Compound 23 exhibited activity at a concentration of 6.25 and $12.5 \mu \mathrm{g} / \mathrm{mL}$ against $B$. pumilus and K. pneumoniae, respectively. Altogether, in these compounds, SARs revealed that the antibacterial activity improved by replacing the benzoic acid substituent with a salicylic acid substituent. Further, compounds containing 4-methyl substituent showed superior activity than the corresponding 4-chloromethyl group bearing compounds (compounds 9-11 vs. compounds 12-14). Interestingly, compounds 21 and 23 containing heteroaromatic ring and chloromethyl group as a substituent at the fourth position of coumarin ring were found to be more active than the corresponding 4-methyl group-containing compounds 20 and 22.

The synthesized compounds were also evaluated against three fungal strains $C$. albicans, $C$. tropicalis, and $A$. niger. The results are summarized in Table 2 and Fig. 3. For C. albicans, MIC values of these compounds vary from 6.25 to $200 \mu \mathrm{g} / \mathrm{mL}$. Among these, compounds 9, 11, 21, and 
Table 1: Antibacterial activity of compounds 1-6 expressed as a minimum inhibitory concentration $(\mu \mathrm{g} / \mathrm{mL})$

\begin{tabular}{|c|c|c|c|c|c|c|c|}
\hline Bacterial strain & Compound 1 & Compound 2 & Compound 3 & Compound 4 & Compound 5 & Compound 6 & Ery $^{a}$ \\
\hline Bs & 200 & 100 & 100 & 100 & 100 & 50 & $<3.25$ \\
\hline $\mathrm{Sa}$ & 200 & 200 & 100 & $50-100$ & 50 & 50 & 2.75 \\
\hline $\mathrm{Bp}$ & 200 & 100 & 100 & 100 & 100 & 25 & 1.5 \\
\hline Kp & $>200$ & $>200$ & $>200$ & $>200$ & 200 & 100 & $<0.39$ \\
\hline St & 200 & 100 & 100 & 100 & 25 & 25 & 6.78 \\
\hline
\end{tabular}

${ }^{a}$ Erythromycin

Table 2: Antimicrobial activities of 7-amidocoumarins as expressed in minimum inhibitory concentration $(\mu \mathrm{g} / \mathrm{mL})$

\begin{tabular}{|c|c|c|c|c|c|c|c|c|c|}
\hline \multirow[t]{2}{*}{ Compound number } & \multicolumn{3}{|c|}{ Gram-positive } & \multicolumn{3}{|c|}{ Gram-negative } & \multirow[t]{2}{*}{$\mathrm{Ca}^{\mathrm{a}}$} & \multirow[t]{2}{*}{$\mathrm{Ct}^{\mathrm{a}}$} & \multirow[t]{2}{*}{$\mathbf{A n}^{\mathrm{a}}$} \\
\hline & $B s^{a}$ & $\mathbf{S a}^{\mathrm{a}}$ & $B p^{a}$ & $E c^{a}$ & $K p^{\mathbf{a}}$ & $\mathbf{S t}^{\mathrm{a}}$ & & & \\
\hline 9 & 25 & 100 & 12.5 & 200 & 12.5 & 200 & 12.5 & 25 & 6.25 \\
\hline 10 & 50 & 100 & 50 & $>200$ & 25 & 100 & 100 & $>200$ & 200 \\
\hline 11 & 6.25 & 50 & 12.5 & 50 & 12.5 & 25 & 6.25 & 6.25 & 25 \\
\hline 12 & 100 & 100 & 25 & 200 & 200 & 50 & 100 & $>200$ & 200 \\
\hline 13 & 100 & 200 & 100 & 100 & $>200$ & $>200$ & 200 & $>200$ & 200 \\
\hline 14 & 50 & 100 & 50 & $>200$ & 100 & 25 & 200 & 100 & 100 \\
\hline 15 & 200 & $>200$ & 50 & 100 & 200 & 50 & 200 & $>200$ & 200 \\
\hline 16 & 200 & 200 & 200 & $>200$ & $>200$ & 200 & 200 & $>200$ & 200 \\
\hline 17 & 100 & $>200$ & 200 & 100 & 200 & 100 & 200 & 100 & 200 \\
\hline 18 & 100 & $>200$ & 200 & 100 & 200 & 50 & 200 & $>200$ & 200 \\
\hline 19 & 50 & 100 & 200 & 200 & 200 & 100 & 200 & $>200$ & 200 \\
\hline 20 & 200 & 50 & 100 & 200 & 100 & 200 & 200 & $>200$ & 100 \\
\hline 21 & 12.5 & 6.25 & 6.25 & 100 & 50 & 6.25 & 12.5 & 25 & 50 \\
\hline 22 & 100 & 100 & 200 & $>200$ & $>200$ & 100 & 50 & 200 & 200 \\
\hline Amp & - & - & - & - & - & - & 6.25 & 12.5 & 6.25 \\
\hline
\end{tabular}

${ }^{a}$ Bacillus subtilis (Bs, MTCC 121), Staphylococcus aureus (Sa, MTCC 96), Bacillus pumilus (Bp, MTCC 1607), Escherichia coli (Ec, MTCC1304), Klebsiella pneumonia (Kp, MTCC 3384), Salmonella typhi (St, MTCC 537), Candida albicans (Ca, MTCC 3017), Candida tropicalis (Ct, MTCC 184), Aspergillus niger (An, MTCC 1344)

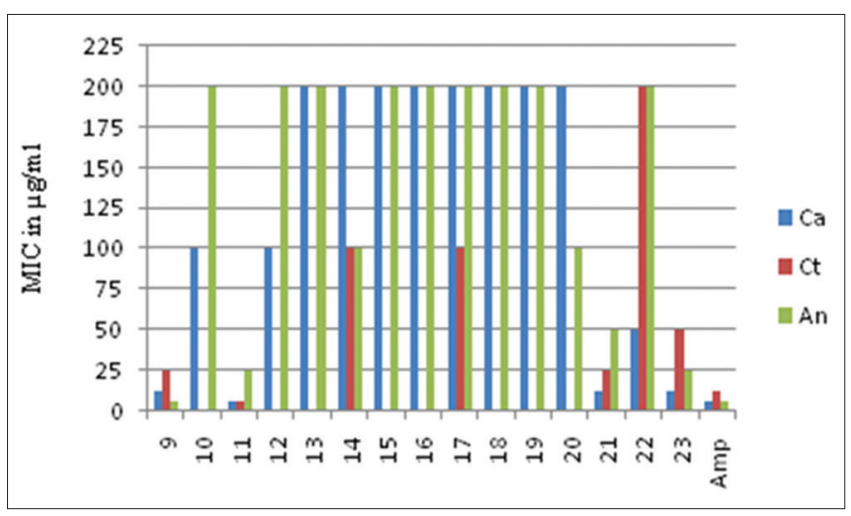

Fig. 3: Antifungal activity of amidocoumarins

23 showed the highest activity (6.25-50 $\mu \mathrm{g} / \mathrm{mL})$. Compound 11 was equipotent to Amp against $C$. albicans and exhibited two-fold superior activity against $C$. tropicalis than the standard drug Amp. The MICs of these four compounds against $A$. niger were found to be in the range of $6.25-50 \mu \mathrm{g} / \mathrm{mL}$. Compound 9 showed promising activity $(6.25 \mu \mathrm{g} / \mathrm{mL})$ against $A$. niger.

\section{Chitinase Inhibitory activity}

Chitin, the linear polymer of $N$-acetylglucosamine, is an essential structural component of the fungal cell wall. Fungal chitinases play an important role in exogenous chitin decomposition, fungal cell wall degradation, and remodeling. These enzymes have been proposed as an important target for the design and development of novel antifungals [24]. Several chitinase inhibitors have been reported in literature, but none of them has yet successfully reached the clinic for the treatment of fungal infections $[25,26]$. Therefore, there is an urgent need for the identification of novel chitinase inhibitors. In this study, the coumarin derivatives 9-23 were also tested for their anti-chitinase activity. Chitinase activity of the compounds was determined by DNS, as mentioned by Miller using colloidal chitin as a substrate [22]. Here, the concept lies in DNS color change from yellowish orange to brownish orange. During the reaction, chitinase breaks colloidal chitin into $\mathrm{N}$-acetyl glucosamine (NAG), a reducing sugar liberated from the hydrolysis of chitin polymer. This reducing sugar binds with DNS to impart a brown color to the solution. If the compound is inhibitory in nature toward chitinase the basic color of DNS does not change and NAG is not formed but if the compound is noninhibitory in nature; it does not interfere in the normal reaction between chitinase and colloidal chitin and change in color is reflected. In our study, compounds 9, 16, 18, 21, and 23 were found to inhibit the enzyme chitinase because, after incubation with these compounds, the enzyme became ineffective to breakdown the colloidal chitin and the basic color of DNS did not change. However, the other coumarin compounds of the series exhibited non-inhibitory behavior toward the chitinases, i.e., the compounds did not interfere in the normal reaction of chitinase with colloidal chitin and color change was observed. Among the tested compounds, compound 9 was found to be the most effective chitinase inhibitor.

\section{CONCLUSION}

In summary, we synthesized a series of 7-benzamidocoumarins and reported their antimicrobial activity for the first time. Some of these 
compounds showed moderate to excellent in vitro activities against different Gram-positive, Gram-negative bacterial strains, and fungi with MIC values between 6.25 and $50 \mathrm{mg} / \mathrm{mL}$. Compound 21 was found to be the most active with MIC $6.25 \mu \mathrm{g} / \mathrm{mL}$ against B. pumilus, S. aureus, and $S$. typhi. Compound 21 also inhibited the growth of fungi $C$. albicans and $C$. tropicalis at concentration 12.5 and $25 \mu \mathrm{g} / \mathrm{mL}$, respectively. The other promising antibacterial molecules from this series were compounds 9, 11, and 23. These compounds showed broad-spectrum activity against both bacterial and fungal strains. Compound 9 emerged as best chitinase inhibitor of the series. Our findings suggest that the further derivatization of the amidocoumarin scaffold could give rise to structures with enhanced antimicrobial and anti-chitinase activities.

\section{ACKNOWLEDGMENTS}

DK thanks Science and Engineering Research Board, India (grant number: EMR/2016/001396) for financial assistance.

\section{AUTHOR'S CONTRIBUTIONS}

DK and VS conceived and planned the experiments. NT and Priyanka synthesized the compounds. VS carried out biological experiments. DK analyzed the data and wrote the manuscript with input from VS.

\section{CONFLICTS OF INTEREST}

The authors declare that they have no conflicts of interest.

\section{REFERENCES}

1. Peng XM, Cai GX, Zhou CH. Recent developments in azole compounds as antibacterial and antifungal agents. Curr Top Med Chem 2013;13:1963-2010.

2. Rai J, Randhawa GK, Kaur M. Recent advances in antibacterial drugs. Int J Appl Basic Med Res 2013;3:3-10.

3. Simmons KJ, Chopra I, Fishwick CW. Structure-based discovery of antibacterial drugs. Nat Rev Microbiol 2010;8:501-10.

4. Jayanthi CR, Chaithra KN, Reddy NS. A profile of adverse drug reactions to antimicrobial agents at a tertiary care hospital. Indian J Pharm Pharmacol 2017;4:16-21.

5. Saeed AM. Pharmaco bacteriological investigation of erythromycin, cephalexin, cephradine, amoxycillin and ciprofloxacin against different strains of Klebsiella pneumonaie, Escherichia coli, Pseudomonas aeruginosa, Staphylococcus aureus and Salmonella species. Indian J Pharm Pharmacol 2018;5:153-8.

6. Walsh C. Molecular mechanisms that confer antibacterial drug resistance. Nature 2000;406:775-81.

7. Denning DW, Bromley MJ. Infectious disease. How to bolster the antifungal pipeline. Science 2015;347:1414-6.

8. Badiee P, Hashemizadeh Z. Opportunistic invasive fungal infections: Diagnosis \& clinical management. Indian J Med Res 2014;139:195-204.

9. Brown GD, Denning DW, Gow NA, Levitz SM, Netea MG, White TC. Hidden killers: Human fungal infections. Sci Transl Med 2012;4:165rv13.
10. Riveiro ME, De Kimpe N, Moglioni A, Vázquez R, Monczor F, Shayo C, et al. Coumarins: Old compounds with novel promising therapeutic perspectives. Curr Med Chem 2010;17:1325-38.

11. Borges F, Roleira F, Milhazes N, Santana L, Uriarte E. Simple coumarins and analogues in medicinal chemistry: Occurrence, synthesis and biological activity. Curr Med Chem 2005;12:887-916.

12. Hoult JR, Payá M. Pharmacological and biochemical actions of simple coumarins: Natural products with therapeutic potential. Gen Pharmacol 1996;27:713-22.

13. Barot KP, Jain SV, Kremer L, Singh S, Ghate MD. Recent advances and therapeutic journey of coumarins: Current status and perspectives. Med Chem Res 2015;24:2771-98.

14. Gaudino EC, Tagliapietra S, Martina K, Palmisano G, Cravotto G. Recent advances and perspectives in the synthesis of bioactive coumarins. RSC Adv 2016;6:46394-405.

15. Singh LK, Priyanka, Singh V, Katiyar D. Design, synthesis and biological evaluation of some new coumarin derivatives as potential antimicrobial agents. Med Chem 2015;11:128-34.

16. Misra S, Singh LK, Priyanka, Gupta J, Misra-Bhattacharya S, Katiyar D. Synthesis and biological evaluation of 4-oxycoumarin derivatives as a new class of antifilarial agents. Eur J Med Chem 2015;94:211-7.

17. Whittemore M, Guillon NH, McNeel T, Rapp R, Mariano T, Heck D, Laskin J. Synthetic approaches to 4,8-dimethyl-4'-(N-pyridiniummethyl)-4',5'-dihydropsoralens and their activity against PAM 212 keratinocytes. Heterocycles 2001;55:1081-94.

18. Kathuria A, Priya N, Chand K, Singh P, Gupta A, Jalal S, et al. Substrate specificity of acetoxy derivatives of coumarins and quinolones towards calreticulin mediated transacetylation: Investigations on antiplatelet function. Bioorg Med Chem 2012;20:1624-38.

19. Stefanachi A, Favia AD, Nicolotti O, Leonetti F, Pisani L, Catto M, et al. Design, synthesis, and biological evaluation of imidazolyl derivatives of 4,7-disubstituted coumarins as aromatase inhibitors selective over 17- $\alpha$-hydroxylase/C17-20 lyase. J Med Chem 2011;54:1613-25.

20. Priyanka, Neelabh, Tiwari N, Sharma RK, Gupta P, MisraBhattacharya S, et al. Synthesis, structure elucidation, homology modeling and antifilarialactivity of 7-benzamidocoumarin derivatives. Chem Select 2019;4:3300-7.

21. Vipra A, Desai SN, Junjappa RP, Roy P, Poonacha N, Ravinder P, et al. Determining the minimum inhibitory concentration of bacteriophages: Potential advantages. Adv Microbiol 2013;3:181-90.

22. Gangwar M, Singh V, Pandey AK, Tripathi CK, Mishra BN. Purification and characterization of chitinase from Streptomyces violascens NRRL B2700. Indian J Exp Biol 2016;54:64-71.

23. National Committee for Clinical Laboratory Standards. Approved Standard Document. M27-A2, Reference Method for Broth Dilution Antifungal Susceptibility Testing of Yeasts. Wayne, PA: National Committee for Clinical Laboratory Standards; 2002.

24. Hartl L, Zach S, Seidl-Seiboth V. Fungal chitinases: Diversity, mechanistic properties and biotechnological potential. Appl Microbiol Biotechnol 2012;93:533-43.

25. Dickinson K, Keer V, Hitchcock CA, Adams DJ. Chitinase activity from Candida albicans and its inhibition by allosamidin. J Gen Microbiol 1989;135:1417-21.

26. Houston DR, Synstad B, Eijsink VG, Stark MJ, Eggleston IM, van Aalten DM. Structure-based exploration of cyclic dipeptide chitinase inhibitors. J Med Chem 2004;47:5713-20. 